\title{
BMJ Open Hypertensive disorders of pregnancy and risk of neurodevelopmental disorders in the offspring: a systematic review and meta-analysis protocol
}

\author{
Gillian M Maher, ${ }^{1,2}$ Gerard W O'Keeffe, ${ }^{1,3}$ Louise C Kenny, ${ }^{1,4}$ Patricia M Kearney, ${ }^{2}$ \\ Ted G Dinan, ${ }^{5,6}$ Ali S Khashan ${ }^{1,2}$
}

To cite: Maher GM, 0'Keeffe GW, Kenny LC, et al. Hypertensive disorders of pregnancy and risk of neurodevelopmental disorders in the offspring: a systematic review and metaanalysis protocol. BMJ Open 2017;7:e018313. doi:10.1136/ bmjopen-2017-018313

- Prepublication history and additional material for this paper are available online. To view these files, please visit the journal online (http://dx.doi org/10.1136/bmjopen-2016018313).

Received 21 June 2017 Revised 9 August 2017 Accepted 30 August 2017

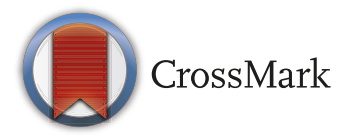

For numbered affiliations see end of article.

Correspondence to

Dr Ali S Khashan;

a.khashan@ucc.ie

\section{ABSTRACT}

Introduction Hypertensive disorders of pregnancy (HDPs), that is chronic hypertension, gestational hypertension, pre-eclampsia (de novo or superimposed on chronic hypertension) and white coat hypertension, affect approximately $5 \%-15 \%$ of pregnancies. HDP exposure has been linked to an increased risk of autism spectrum disorder, attention deficit/hyperactivity disorder and other neurodevelopmental disorders in children. However, findings are inconsistent, and a clear consensus on the impact of HDPs on the risk of neurodevelopmental disorders is needed. Therefore, we aim to synthesise the published literature on the relationship between HDPs and the risk of neurodevelopmental disorders in the form of a systematic review and meta-analysis.

Methods and analysis We will include cohort, casecontrol and cross-sectional studies in which diagnosis of an HDP was reported, and neurodevelopmental disorders were the outcome of interest based on a preprepared protocol. A systematic search of PubMed, CINAHL, Embase, PsycINF0 and Web of Science will be conducted in accordance with a detailed search strategy. Two authors will independently review the titles and abstracts of all studies, perform data extraction using a standardised data collection form and assess study quality using a bias classification tool. Meta-analyses will be performed to calculate overall pooled estimates using the generic inverse variance method. This systematic review will be reported according to the Preferred Reporting Items for Systematic reviews and Meta-Analyses.

Ethics and dissemination This proposed systematic review and meta-analysis is based on published data, therefore, does not require ethics approval. Findings will be presented at scientific conferences and disseminated through publication in a peer-reviewed journal. Registration CRD42017068258.

\section{INTRODUCTION}

Hypertensive disorders of pregnancy (HDPs) are the most common complications of pregnancy estimated to affect approximately $5 \%-15 \%$ of all pregnancies. ${ }^{12}$ HDPs are classified into four categories, as recommended by the International Society for the Study

\section{Strengths and limitations of this study}

- The proposed systematic review and meta-analysis will adhere to the Preferred Reporting Items for Systematic Reviews and Meta Analyses guidelines, ensuring consistency and uniformity in reporting the full systematic review.

- The review aims to provide a clear consensus on the relationship between hypertensive disorders of pregnancy and neurodevelopmental disorders in the offspring.

- Two reviewers will screen for study eligibility and perform the quality assessment, minimising the likelihood of reviewer-based bias in the systematic review.

- A limitation of the review is that it will only include the published literature in the English language.

of Hypertension in Pregnancy": 'chronic hypertension', 'gestational hypertension', 'pre-eclampsia-de novo or superimposed on chronic hypertension' and 'white coat hypertension'. While HDPs are not fully understood, risk factors include advanced maternal age and elevated body mass index, both of which are increasingly common in modern society. ${ }^{4}$ HDP create a hostile in utero environment as a result of multiple pathophysiological changes including reduced placental blood flow, maternal inflammation and oxidative stress. ${ }^{5}$ These can potentially alter fetal developmental trajectories, which may increase the risk of long-term vascular, cognitive and psychiatric sequelae in the offspring. ${ }^{4-8}$

Neurodevelopmental disorders including autism spectrum disorder (ASD) and attention deficit/hyperactivity disorder (ADHD) are a group of conditions with onset during the developmental period and may lead to impairments in personal, social, academic or occupational functioning. ${ }^{9} 10$ Though 
these disorders have a strong genetic basis, ${ }^{11} 12$ there is increasing evidence suggesting that environmental risk factors during prenatal development may also play a role. ${ }^{11}{ }^{13-18}$ In support of this, a population-based study conducted on a Swedish population estimated that genes and environmental exposure each contribute approximately half of the overall risk of ASD, with this $50 / 50$ contribution remaining consistent across the study's 24-year span. ${ }^{11}$ Furthermore, recent work demonstrated focal patches of abnormal laminar architecture and laminar disorganisation in the prefrontal and temporal cortices of children with ASD suggesting there may be alterations in brain development at prenatal stages, as cortical lamination is ongoing during the second trimester of pregnancy. ${ }^{19}{ }^{20}$ Moreover, there is some evidence for alterations in brain structural and vascular anatomy $^{21}$ and reduced cognitive functioning ${ }^{22}$ in offspring of pregnancies complicated by pre-eclampsia pregnancies. There is therefore a need to determine the impact of HDP exposure on the risk of adverse neurodevelopmental outcomes in the children.

\section{Early identification and intervention}

There is a growing consensus that early identification and intervention are key to improving long-term neurodevelopmental outcomes. ${ }^{23}{ }^{24}$ Previously published work has indicated that early behavioural intervention if commenced before 30 months old, can lead to improvements in cognitive and adaptive behaviour among individuals with ASD. ${ }^{25} 26$ Despite this increasing recognition for surveillance, the average age of ASD diagnosis remains at approximately $4-5$ years, meaning the window for intervention has closed. ${ }^{23} 2728$ However, research suggests that a stable diagnosis can be made as young as 2 years, allowing earlier access to specialised services. ${ }^{29}$ Therefore, by examining the potential impact of HDP on neurodevelopment in offspring, it can inform the need for increased paediatric surveillance of infants who have been exposed to HDP. This in turn could allow for early intervention that may aid improvement of neurodevelopmental outcome. ${ }^{23252630}$

\section{Rationale for current systematic review}

Evidence suggests that HDP may lead to an increased risk of ASD, ADHD as well as other neurodevelopmental disorders in children. ${ }^{15} 3132$ Conversely, other studies have reported no association, ${ }^{13} 31$ highlighting the need for further study in this area. Therefore, the aim of this systematic review and meta-analysis is to summarise the available evidence examining the association between pre-eclampsia and gestational hypertension, and subsequent risk of neurodevelopmental disorders in exposed children, and if possible to identify an overall pooled estimate of association. The systematic review will be based on the following requirements:

\section{Population}

Pregnant women and their children.
Intervention/exposures

HDP.

Primary exposure: pre-eclampsia.

Secondary exposure: other HDP.

Comparison

No diagnosis of HDP.

\section{Outcomes}

Primary outcome 1: ASD.

Primary outcome 2: ADHD.

Secondary outcomes: other neurodevelopmental disorders.

\section{METHODS AND DESIGN}

This systematic review and meta-analysis will follow the Preferred Reporting Items for Systematic Reviews and Meta Analyses (PRISMA) guidelines. ${ }^{33}$

\section{OBJECTIVES}

This study aims to conduct a systematic review and meta-analysis to examine the association between HDP and neurodevelopmental disorders in the offspring.

\section{REVIEW QUESTION}

This systematic review will address the following research question:

What are the pooled estimates from existing literature examining the association between HDP and neurodevelopmental disorders in the offspring?

\section{CRITERIA FOR CONSIDERING STUDIES FOR THE REVIEW Inclusion criteria}

- We will include cohort, case-control or cross-sectional studies in which a diagnosis of HDP was reported and neurodevelopmental disorders are the outcome of interest.

- Examining the association between HDP and neurodevelopmental disorders must be part of the main objective of the study. (This includes studies that aimed to look at other perinatal risk factors in addition to HDP.)

- Data must be from an original study, and HDP may be confirmed through medical records or doctor-diagnosed self-reporting.

- We will include studies published in English only, including all years from inception of the electronic databases until June 2017.

- Peer-reviewed literature only will be included.

- Neurodevelopmental and other behavioural or cognitive outcomes will be the focus of this review. Motor disorders have been included in the search strategy to capture studies that have included these outcomes.

Exclusion criteria

- Studies that are not in English. 
- Studies where the participants are not human.

- Case reports, case series, letters, commentaries, notes, editorials and conference abstracts.

\section{Search strategy for identifying relevant studies Bibliographic database searches}

1. One reviewer (GMM) will conduct a systematic search of the literature in the following electronic databases: PubMed, CINAHL, Embase, PsycINFO and Web of Science. A detailed search strategy has been compiled, and these terms will be searched according to the principles of Boolean Logic (AND, OR and NOT) and using Medical Subject Headings. For example, ('Pre-eclampsia' OR 'gestational hypertension') AND ('autism spectrum disorder' OR 'attention deficit/ hyperactivity disorder' OR 'neurodevelopmental disorder'). (The full search strategy is included in online supplementary file 1 ).

2. Searches of the electronic databases will be supplemented by hand-searching the reference lists of included studies for further potentially eligible studies.

\section{Selection of studies for inclusion in the review}

Titles and abstracts of studies retrieved from each database search will be stored and managed in EndNote reference manager. Two review authors (GMM and ASK) will independently review the titles and abstracts of all studies. Full texts will be obtained where necessary to screen for eligibility in the systematic review and meta-analysis in accordance with the predefined inclusion/exclusion criteria. Where consensus on eligibility cannot be achieved, a third review author (GWOK) will be involved in the discussion.

\section{Data extraction and management}

Using a standardised data collection form, two reviewers (GMM and GWOK) will independently extract data from the eligible studies including the author and year of publication, study design, definition of exposure and outcome used, sample size, confounders adjusted for (if any) as well as crude and adjusted estimates. Discrepancies will be resolved by a third reviewer (ASK) if necessary.

\section{Appraisal of the quality of included studies}

Quality assessment of the included studies will be conducted by two reviewers (GMM and PMK) independently and agreed on subsequently using an appropriate quality assessment tool depending on the study design. Discrepancies will be resolved by a third reviewer (ASK) if necessary. A bias classification tool described in detail elsewhere will be used. ${ }^{34}$ In summary, this tool uses a checklist to assess common features of the six types of bias most often associated with observational studies (selection, exposure, outcome, analytic, attrition and confounding). Study bias is then classified as minimal, low, moderate, high or not reported for each of the six types of bias and an overall likelihood of bias based on the total of the six types of bias will be measured and reported. For example, selection bias will be minimised if the sample was taken from a 'consecutive unselected population', while conversely a study with high selection bias will arise if sample selection is ambiguous and the sample is not likely representative.

\section{Data synthesis including assessment of heterogeneity}

Where the data allow, meta-analyses will be performed to calculate overall pooled estimates of the relationship between combined HDP, pre-eclampsia and gestational hypertension, and different disorders of neurodevelopment. Both crude and adjusted results will be displayed where possible using the generic inverse variance method. Adjustment will be based on the definition outlined in each identified study and a hierarchy of adjustment created depending on the factors that are adjusted for. Studies that report similar adjustments will be analysed separately in crude and adjusted models to assess potential confounding among studies that reported adjusted estimates.

A fixed-effects model will be used where heterogeneity is low $\left(\mathrm{I}^{2}\right.$ value of less than $\left.50 \%\right)$, and a random-effects model where heterogeneity is high $\left(\mathrm{I}^{2}\right.$ value of $50 \%$ or more) according to the Cochrane Handbook criteria. ${ }^{35}$

We will also perform the following subgroup/sensitivity analyses where the data allow, using RevMan 5.3:

1. according to study design (cohort vs case-control vs cross-sectional)

2. according to studies that report estimates for the association between pre-eclampsia and gestational hypertension and each neurodevelopmental disorder

3. according to location (eg, Europe vs USA)

4. according to income level of country (low/middle/ high)

5. according to study quality (minimal/low vs moderate/high)

6. according to measurement of exposure and outcome data (self-reported vs medical records based on varying clinical coding systems).

Publication bias will be assessed using a funnel plot provided at least 10 or more studies are included in the meta-analysis. The trim and fill method will also be used to identify and correct for funnel plot asymmetry arising from publication bias. ${ }^{36}$ Where any other subgroup/ sensitivity analyses are identified in the process of the meta-analysis, such as analyses to explore potential high heterogeneity or publication bias, these will be clearly labelled as post hoc analyses.

\section{Presenting and reporting the results}

A flow diagram (as outlined in the PRISMA statement $t^{33}$ ) will be included to outline the study selection process step by step, and a rationale provided for excluded studies. The characteristics and quality assessment of the included studies will be presented in tables. Pooled estimates will be presented using forest plots. Where a study is eligible for inclusion in the systematic review but does not provide adequate data to include in a meta-analysis, 
we will contact the corresponding authors in an attempt to obtain raw data where appropriate. If raw data cannot be obtained, the findings will be included individually in a separate table.

\section{CONCLUSION}

This systematic review and meta-analysis will summarise existing literature examining the association between HDP and different disorders of neurodevelopment based on a preprepared protocol. By identifying the possible contributors to adverse neurodevelopmental outcomes, it may lead to early identification and intervention. Therefore, by examining potential aetiologies of neurodevelopmental disorders, it may inform the need for greater paediatric surveillance of HDP-exposed infants to allow early intervention, which may aid improvement of neurodevelopmental outcome. ${ }^{23252630}$

\section{Potential limitations}

It is anticipated that publication bias may pose as a limitation for this review. Studies that show an effect have an increased likelihood of being published as well as being published in English. Due to limited resources, the systematic review search will be confined to studies published in the English language only, potentially resulting in publication bias as well as relevant indexed studies being overlooked. If possible, a funnel plot will be used to assess the presence of publication bias.

Furthermore, the presence of confounding is a major concern in observational studies. Potential confounders may include infant sex, family's socioeconomic status, ethnicity, maternal age, parity, maternal smoking and alcohol status during pregnancy, maternal use of antidepressants (during pregnancy or during the preconception period) and maternal mental illness, while preterm delivery and small for gestational age could potentially play a confounding or mediating role. As mentioned above, our meta-analyses will display both crude and adjusted results where possible using the generic inverse variance method, basing adjustment on the definition outlined in each identified study.

\section{Ethics and dissemination}

Given that this is a protocol for a systematic review and based on published data, there is no requirement for ethics approval. It is anticipated that dissemination of results will take place at conferences and through publication in a peer-reviewed journal.

\footnotetext{
Author affiliations

${ }^{1}$ The Irish Centre for Fetal and Neonatal Translational Research (INFANT), Cork

University Maternity Hospital and University College Cork, Cork, Ireland

${ }^{2}$ Department of Epidemiology and Public Health, Western Gateway Building, University College Cork, Cork, Ireland

${ }^{3}$ Department of Anatomy and Neuroscience, Western Gateway Building, University College Cork, Cork, Ireland

${ }^{4}$ Department of Obstetrics and Gynaecology, Cork University Maternity Hospital, University College Cork, Cork, Ireland
}

${ }^{5}$ Department of Psychiatry, Cork University Hospital and University College Cork, Cork, Ireland

${ }^{6}$ APC Microbiome Institute, University College Cork, Cork, Ireland

Contributors GMM and ASK conceived and designed the protocol, and GMM drafted the protocol manuscript. GMM and ASK participated in the development of the search strategy. GMM and GWOK planned the data extraction. GMM and PMK planned quality appraisal of included studies. GWOK, LCK, PMK, TGD and ASK critically revised the manuscript for methodological and intellectual content. All authors approved the final version.

Funding This publication has emanated from research conducted with the financial support of the Health Research Board (HRB), Ireland under the SPHeRE (Structured Population andHealth-services Research Education) Programme and from Science Foundation Ireland (SFI) in the form of a research centre grant to the Irish Centre For Fetal and Neonatal Translational Research under the grant number INFANT-12/RC/2272. The Alimentary Pharmabiotic Centre (APC) is a research centre also funded by SFI under the grant number SFI/12/RC/2273.

Competing interests None declared.

Provenance and peer review Not commissioned; externally peer reviewed.

Open Access This is an Open Access article distributed in accordance with the Creative Commons Attribution Non Commercial (CC BY-NC 4.0) license, which permits others to distribute, remix, adapt, build upon this work non-commercially, and license their derivative works on different terms, provided the original work is properly cited and the use is non-commercial. See: http://creativecommons.org/ licenses/by-nc/4.0/

(c) Article author(s) (or their employer(s) unless otherwise stated in the text of the article) 2017. All rights reserved. No commercial use is permitted unless otherwise expressly granted.

\section{REFERENCES}

1. Olson-Chen C, Seligman NS. Hypertensive Emergencies in Pregnancy. Crit Care Clin 2016;32:29-41.

2. Gillon TE, Pels A, von Dadelszen P, et al. Hypertensive disorders of pregnancy: a systematic review of International clinical practice guidelines. PLoS One 2014;9:e113715.

3. Tranquilli AL, Dekker G, Magee L, et al. The classification, diagnosis and management of the hypertensive disorders of pregnancy: A revised statement from the ISSHP. Pregnancy Hypertens 2014;4:97-104.

4. Hakim J, Senterman MK, Hakim AM. Preeclampsia is a biomarker for vascular disease in both mother and child: the need for a medical alert system. Int J Pediatr 2013;2013:1-8.

5. Chaiworapongsa T, Chaemsaithong P, Yeo L, et al. Pre-eclampsia part 1: current understanding of its pathophysiology. Nat Rev Nephrol 2014;10:466-80.

6. Davis EF, Lazdam M, Lewandowski AJ, et al. Cardiovascular Risk Factors in Children and Young Adults Born to Preeclamptic Pregnancies: A Systematic Review. Pediatrics 2012;129:e155 2-e1561.

7. Nomura Y, John RM, Janssen AB, et al. Neurodevelopmental consequences in offspring of mothers with preeclampsia during pregnancy: underlying biological mechanism via imprinting genes. Arch Gynecol Obstet 2017;295:1319-29.

8. Pinheiro TV, Brunetto S, Ramos JG, et al. Hypertensive disorders during pregnancy and health outcomes in the offspring: a systematic review. J Dev Orig Health Dis 2016;7:391-407.

9. Lai MC, Lombardo MV, Baron-Cohen S. Autism. Lancet 2014;383:896-910.

10. Thapar A, Cooper M. Attention deficit hyperactivity disorder. Lancet 2016;387.

11. Sandin S, Lichtenstein P, Kuja-Halkola $R$, et al. The familial risk of autism. JAMA 2014;311:1770-7.

12. Akutagava-Martins GC, Rohde LA, Hutz MH. Genetics of attentiondeficit/hyperactivity disorder: an update. Expert Rev Neurother 2016;16:145-56.

13. Gardener H, Spiegelman D, Buka SL. Prenatal risk factors for autism: comprehensive meta-analysis. Br J Psychiatry 2009;195:7-14.

14. Krakowiak $P$, Walker CK, Bremer AA, et al. Maternal metabolic conditions and risk for autism and other neurodevelopmental disorders. Pediatrics 2012;129:e1121-e1128.

15. Mann JR, McDermott S, Bao H, et al. Pre-eclampsia, birth weight, and autism spectrum disorders. J Autism Dev Disord 2010;40:548-54. 
16. Mann JR, McDermott S. Are maternal genitourinary infection and pre-eclampsia associated with ADHD in school-aged children? J Atten Disord 2011;15:667-73.

17. Silva D, Colvin L, Hagemann E, et al. Environmental risk factors by gender associated with attention-deficit/hyperactivity disorder. Pediatrics 2014;133:e14-e22.

18. Walker CK, Krakowiak P, Baker A, et al. Preeclampsia, placental insufficiency, and autism spectrum disorder or developmental delay. JAMA Pediatr 2015;169:154-62.

19. Stiles J, Jernigan TL. The basics of brain development. Neuropsychol Rev 2010;20:327-48.

20. Stoner R, Chow ML, Boyle MP, et al. Patches of disorganization in the neocortex of children with autism. $N$ Engl J Med 2014;370:1209-19.

21. Rätsep MT, Paolozza A, Hickman AF, et al. Brain Structural and Vascular Anatomy Is Altered in Offspring of Pre-Eclamptic Pregnancies: A Pilot Study. Am J Neuroradiol 2016;37.

22. Rätsep MT, Hickman AF, Maser B, et al. Impact of preeclampsia on cognitive function in the offspring. Behav Brain Res 2016;302:175-81.

23. Zwaigenbaum L, Bauman ML, Stone WL, et al. Early Identification of Autism Spectrum Disorder: Recommendations for Practice and Research. Pediatrics 2015;136:S10-S40.

24. Myers SM, Johnson CP. American Academy of Pediatrics Council on Children With Disabilities. Management of children with autism spectrum disorders. Pediatrics 2007;120:1162-82.

25. Dawson G, Rogers S, Munson J, et al. Randomized, controlled tria of an intervention for toddlers with autism: the Early Start Denver Model. Pediatrics 2010;125:e17-e23.

26. Dawson G, Jones EJ, Merkle K, et al. Early behavioral intervention is associated with normalized brain activity in young children with autism. J Am Acad Child Adolesc Psychiatry 2012;51:1150-9.
27. Fountain C, King MD, Bearman PS. Age of diagnosis for autism: individual and community factors across 10 birth cohorts. $J$ Epidemiol Community Health 2011;65:503-10.

28. Mandell DS, Morales $\mathrm{KH}$, Xie M, et al. Age of diagnosis among Medicaid-enrolled children with autism, 2001-2004. Psychiatr Serv 2010;61:822-9.

29. Lord C, Risi S, DiLavore PS, et al. Autism from 2 to 9 years of age. Arch Gen Psychiatry 2006;63:694-701.

30. Van Hus J, Jeukens-Visser M, Koldewijn K, et al. Early intervention leads to long-term developmental improvements in very preterm infants, especially infants with bronchopulmonary dysplasia. Acta Paediatr 2016;105:773-81.

31. Tuovinen S, Eriksson JG, Kajantie E, et al. Maternal hypertensive pregnancy disorders and cognitive functioning of the offspring: a systematic review. J Am Soc Hypertens 2014;8:832-47.

32. Zhu T, Gan J, Huang J, et al. Association between perinatal hypoxicischemic conditions and attention-deficit/hyperactivity disorder: a meta-analysis. J Child Neurol 2016;31:1235-44.

33. Moher D, Liberati A, Tetzlaff J, et al. Preferred reporting items for systematic reviews and meta-analyses: the PRISMA statement. Ann Intern Med 2009;151:264-9.

34. McDonald SD, Han Z, Mulla S, et al. Preterm birth and low birth weight among in vitro fertilization singletons: a systematic review and meta-analyses. Eur J Obstet Gynecol Reprod Biol 2009;146:138-48.

35. Higgins JPT, Green S. The Cochrane Collaboration, (editors). Cochrane Handbook for Systematic Reviews of Interventions Version 5.1.0 (updated March 2011). 2011. www. handbook.cochrane.org.

36. Duval S. Tweedie R. A Nonparametric "Trim and Fill" Method of Accounting for Publication Bias in Meta-Analysis. J Am Stat Assoc 2000;95:89-98. 